\title{
ON FURTHER CLASSES OF MARTINGALE-LIKE SEQUENCES AND SOME DECOMPOSITION AND CONVERGENCE THEOREMS
}

\author{
DINH QUANG LUU* \\ Hanoi Institute of Mathematics, P.O. Box 631 Bo-Ho, Hanoi, Vietnam \\ Actual address: IM - Stefan Banach Center, ul. Mokotowska 25, skr. 137, 00-950 Warsaw, Poland
}

(Received 23 September, 1997)

\begin{abstract}
It is known that the class of mils generalizes that of pramarts and martingales in the limit. Also every Banach space-valued mil $\left(X_{n}\right)$ with $\lim \inf _{n} E\left(\left\|X_{n}\right\|\right)<\infty$ can be written in a unique form: $X_{n}=M_{n}+P_{n}(n \in \mathbf{N})$, where $\left(M_{n}\right)$ is a uniformly integrable martingale and $\left(P_{n}\right)$ converges to zero a.s. in norm. We shall show that this result still holds for a class which essentially generalizes that of mils. Another class of Banach space-valued martingale-like sequences, still containing all pramarts is defined and shown to have the decomposition above under the following much weaker condition: $\lim \inf _{r \in T} E\left(\left\|X_{\tau}\right\|\right)<\infty$, where $T$ denotes the set of all bounded stopping times.
\end{abstract}

1991 Mathematics Subject Classification. 60G48, 60B11.

0. Definitions and results. Throughout this note, let $(\Omega, \mathcal{A}, P)$ be a complete probability space, $\left(\mathcal{A}_{n}\right)$ an increasing sequence of sub- $\sigma$-algebras of $\mathcal{A}$, and $T$ the set of all bounded stopping times w.r.t. $\left(\mathcal{A}_{n}\right)$. Then $T$ is a directed set with the usual order $(\leq)$, given by $\sigma \leq \tau$ iff $\sigma(\omega) \leq \tau(\omega)$, a.s. Thus the set of all positive integers $\mathbf{N}$ can be regarded as a cofinal subset of $T$. Besides cofinal subsets $U$ of $\mathbf{N}$ and $\Sigma$ of $T$ we shall be dealing with sequences $\left(\tau_{n}\right)$ of $T$ that are always assumed to be increasing and cofinal. We write $\left(\tau_{n}\right) \in \mathcal{T}^{c}$. In particular, if $\tau_{n} \leq \tau_{n+1}^{(-)}(n \in \mathbf{N})$, then $\left(\tau_{n}\right)$ is said to be strongly increasing, where given $\tau \in T$ we denote $\tau^{(-)}=\min \{k \in \mathbf{N}$, $P(\{\tau=k\})>0\}$. To avoid any confusion, we shall denote the set of all elements of $\left(\tau_{n}\right)$ by $\left\{\tau_{n}\right\}$. Further, for simplicity, given a cofinal subset $\Sigma$ of $T, p \in \mathbf{N}$ and $\tau \in T$ with $p \leq \tau$ we use the following notations: $\Sigma(p)=\{\sigma \in \Sigma, p \leq \sigma\}$ and $\Sigma(p, \tilde{\tau})=\{\sigma \in \Sigma, p \leq \sigma \leq \tau\}$. Now let $F$ be a separable Banach space. We shall consider in this note only sequences $\left(X_{n}\right)$ of $F$-valued Bochner integrable functions, defined on $\Omega$ and assumed to be adapted to $\left(\mathcal{A}_{n}\right)$; i.e. each $X_{n}$ is $\mathcal{A}_{n}$-measurable. For other related notions we refer to [5]. Here we recall only the following definition.

Definition 0.1. A sequence $\left(X_{n}\right)$ is said to be

(a) a pramart if for every $\varepsilon>0$ there exists $p \in \mathbf{N}$ such that, for all $\tau \in T(p)$ and $\sigma \in T(p, \tau)$, we have

$$
P(\|D X(\sigma, \tau)\|>\varepsilon)<\varepsilon
$$

where $X_{\sigma}(\tau)$ denotes the $\mathcal{A}_{\sigma}$-conditional expectation of $X_{\tau}$ and $D X(\sigma, \tau)=X_{\sigma}(\tau)-X_{\sigma}$; 
(b) a martingale in the limit if

$$
\limsup _{n} \sup _{m \geq n} D X(n, m) \|=0 \text { a.s.; }
$$

(c) a mil if for every $\varepsilon>0$ there exists $p \in \mathbf{N}$ such that, for all $n \in \mathbf{N}(p)$ we have

$$
P\left(\max _{q \in \mathbf{N}(p, n)}\|D X(q, n)\|>\varepsilon\right)<\varepsilon
$$

(d) a game which becomes fairer with time if for every $\varepsilon>0$ there exists $p \in \mathbf{N}$ such that, for all $m \in \mathbf{N}(p)$ and $n \in \mathbf{N}(p, m)$, we have

$$
P(\|D X(n, m)\|>\varepsilon)<\varepsilon .
$$

Games fairer with time were introduced by L. H. Blake (1970), martingales in the limit by A. G. Mucci (1976), pramarts by A. Millet and L. Sucheston (1980) and mils by M. Talagrand (1985). These classes of martingale-like sequences have been extensively considered by many other authors; e.g. games fairer with time by J. Subramanian in [17] and D. Q. Luu in $[\mathbf{9 , 1 0}]$, martingales in the limit by M Peligrad [15] and J. A. Dvoretzky and A. Bellow in [4], pramarts by L. Egghe in [6] and M. Slaby [16] and mils by Zen-Peng Wang and Xing-Hong Xue in [19], D. Q. Luu [11] etc... It was shown that every one of the aforementioned classes of martingale-like sequences is strictly contained in the next one. For some related examples, see A. Dvoretzky and A. Bellow [4], M. Talagrand [18] and D. Q. Luu [10,11].

The main results we shall prove still hold for the following classes of martingalelike sequences.

Definition 0.2. Let $\Gamma$ be a cofinal subset of $T$. A sequence $\left(X_{n}\right)$ is said to be a $\Gamma$ mil if for every $\varepsilon>0$ there exists $p \in \mathbf{N}$ such that, for all $\gamma \in \Gamma(p)$, we have

$$
P\left(\max _{q \in \mathbf{N}(p, \gamma)}\|D X(q, \gamma)\|>\varepsilon\right)<\varepsilon .
$$

In general if this occurs for some increasing cofinal sequence $\Gamma=\left(\sigma_{n}\right)$ of $\Sigma$, then $\left(X_{n}\right)$ is said to be a $\Sigma$-sequential mil. In particular, every $T$-sequential mil (or $T$-mil, respectively will be called a sequential mil (or universal mil, respectively).

Thus by definition, when $\Sigma$ increases the class of $\Sigma$-sequential mils increases but conversely the class of $\Sigma$-mils decreases. This implies that among the classes of martingale-like sequences introduced above, sequential mils (or universal mils, respectively) form the maximal (or the minimal, respectively) element. Furthermore, $\left(X_{n}\right)$ is a mil if and only if it is an $\mathbf{N}$-mil. Thus, by Example 3.6 of [11], it follows that the class of $\mathbf{N}$-sequential mils not only contains all mils but even differs from that of games fairer with time. Here, we construct the following example.

EXAMPLE 1. There exists a real-valued sequential mil which fails to be either a game fairer with time or an $\mathbf{N}$-sequential mil.

Further, we say that $\left(X_{n}\right)$ is an $L^{1}$-amart w.r.t. $\Sigma$ if for every $\varepsilon>0$ there exists $p \in \mathbf{N}$ such that, for all $\sigma \in \Sigma(p)$ and $\rho \in \Sigma(p, \sigma)$, we have $E(\|D X(\rho, \sigma)\|<\varepsilon)$. In the 
case, when $\Sigma=\mathbf{N}$, M. Peligrad (1976) used this condition to prove the strong a.s. convergence for martingales in the limit taking values in a Banach space with the Radon-Nikodyn property (RNP). Later, we took this condition to define $L^{1}$-amarts and completely characterized all the sequences which have a Riesz decomposition in $[7,8]$. Here, we are interested in Theorem 3.4. of [11] which says that if $\left(X_{n}\right)$ is an $L^{1}$ amart w.r.t. $U$ then $\left(X_{n}\right)$ is a $U$-mil if and only if it can be written in a unique form

$$
X_{n}=M_{n}+P_{n},
$$

where $\left(M_{n}\right)$ is a (not necessarily $L^{1}$-bounded) martingale and $\left(P_{n}\right)$ converges to zero a.s. It is worth noting that this result still holds true if $U$ is replaced by any $\Sigma$. To obtain the characterization results we should consider only the first case, where $\left(X_{n}\right)$ is assumed to be an $L^{1}$-amart w.r.t. $\Sigma$ which guarantees the existence of the martingale $\left(M_{n}\right)$ without the second $L^{1}$-boundedness condition. Then the technique, given by the author in [11], cannot be applied any more to prove the main theorem in this note. Thus we shall return to the classical result in Martingale Theory which says that every $L^{1}$-bounded martingale converges scalarly to zero a.s. and converges (strongly) to zero, a.s. Based on the lemma and its mil version recently obtained by M. Talagrand [18, Theorem 6] we get the following result which is of particular interest.

Theorem 2. Let $\left(X_{n}\right)$ be a $\left\{\sigma_{n}\right\}$-mil for some $\left(\sigma_{n}\right) \in \mathcal{T}^{c}$. Suppose that

$$
\lim \inf _{n} f E \mid\left(\left\|X_{\sigma_{n}}\right\|\right)<\infty
$$

and the sequence $\left(X_{\tau_{n}}\right)$ converges to zero a.s., for some $\left(\tau_{n}\right) \in \mathcal{T}^{c}$. Then $\left(X_{n}\right)$ also converges to zero a.s.

Since every sequence converging in probability contains a subsequence which converges a.s. we obtain the following result.

COROllary 3. Let $\left(X_{n}\right)$ be a sequential mil satisfying

$$
\lim \sup _{\tau \in T} \int_{\Omega}\left\|X_{\tau}\right\| d P<\infty
$$

or a universal mil satisfying

$$
\lim \inf _{\tau \in T} \int_{\Omega}\left\|X_{\tau}\right\| d P<\infty
$$

Suppose that $\left(X_{\tau_{n}}\right)$ converges to zero in probability, for some $\left(\tau_{n}\right) \in \mathcal{T}^{c}$. Then $\left(X_{n}\right)$ converges to zero a.s.

Besides the independent result [11, Theorem 3.4], the main interest of the notion of $\Sigma$-mils is the following result.

TheOREM 4. Let $\left(X_{n}\right)$ be a $\left\{\sigma_{n}\right\}$-mil, for some $\left(\sigma_{n}\right) \in \mathcal{T}^{c}$. Suppose that the condition (1) is satisfied. Then $\left(X_{n}\right)$ can be written in a unique form: 


$$
X_{n}=M_{n}+P_{n}
$$

where $\left(M_{n}\right)$ is a uniformly integrable martingale and $\left(P_{n}\right)$ is a sequential mil which converges to zero a.s.

Consequently, if either the set $\left\{X_{n}(\omega)\right\}$ is relatively weakly compact a.s. or $F$ has the (RNP), then $\left(X_{n}\right)$ converges a.s.

Returning to Condition (1) in Theorem 2 one sees that it depends on the choice of $\left\{\sigma_{n}\right\}$. To avoid this we need the notion of universal mils. But how large is the class of universal mils? The following remark gives a positive answer to the question.

\section{REMARK 5. The class of universal mils contains all pramarts.}

Now, by the remark given after Definition 0.2, we know that every universal mil is a $\Sigma$-mil, for any cofinal subset $\Sigma$ of $T$. It is also known that if $\left(M_{n}\right)$ is a uniformly integrable martingale such that the martingale $\left(M_{\tau_{n}}\right)$ converges weakly a.s., for some $\left(\tau_{n}\right) \in \mathcal{T}^{c}$, then $\left(M_{n}\right)$ also converges a.s. Thus the interest of the main result and of the notions of sequential and universal mils is that, in particular, it allows one to reduce the condition (1) in the case of $\left\{\sigma_{n}\right\}$-mils to Condition (2) or (3) for the case of sequential or universal mils, respectively.

THEOREm 6. Let $\left(X_{n}\right)$ be a sequential or universal mil, respectively. Suppose that the condition (2) or (3) is satisfied. Then $\left(X_{n}\right)$ admits a unique decomposition $X_{n}=M_{n}+P_{n}$, where $\left(M_{n}\right)$ is a uniformly integrable martingale and $\left(P_{n}\right)$ is a sequential or universal mil, respectively, that goes to zero a.s.

Consequently, if the sequence $\left(X_{\tau_{n}}\right)$ converges to an $X: \Omega \rightarrow F$ weakly a.s., for some $\left(\tau_{n}\right) \in \mathcal{T}^{c}$, then the function $X$ is Bochner integrable and $\left(X_{n}\right)$ also converges to $X$ a.s.

Nevertheless, the Talagrand's structure decomposition theorem [18, Theorem 8] is only a particular case of Theorem 4. It is independent from the last theorem, as the following example shows.

EXAMPLE 7. There is a real-valued mil which fails to be a universal mil.

Finally, before going to prove all the aforementioned results, it is worth noting that in both proofs of Theorem 2 and Theorem 4 we essentially use the following simple fact and its consequence.

For every adapted sequence $\left(X_{n}\right)$ of $E$-valued Bochner integrable functions, $p \in \mathbf{N}, \tau \in T(p)$, and a finite sequence $\left\{\tau_{i}, i \leq m\right\}$ of $T\left(p, \tau^{(-)}\right)$we have

$$
\max _{i \leq m}\left\|D X\left(\tau_{i}, \tau\right)\right\| \leq \max _{q \in \mathbf{N}(p, \tau)}\|D X(q, \tau)\|, \text { a.s. }
$$

Consequently, if $\left(X_{n}\right)$ is a $\left\{\sigma_{n}\right\}$-mil, for some strongly increasing sequence $\left(\sigma_{n}\right) \in \mathcal{T}^{c}$, then the sequence $\left(Y_{n}\right)$ defined by $Y_{n}=X_{\sigma_{n}},(n \in \mathbf{N})$, is a mil w.r.t. the increasing sequence $\left(\mathcal{B}_{n}=\mathcal{A}_{\sigma_{n}}\right)$ of sub- $\sigma$-fields of $\mathcal{A}$.

The following last counterexample will be constructed to show that the fact above and its consequence, respectively, fails without the assumption " $\tau_{i} \leq \tau^{(-)}$, $i \leq m$ " and "strongly increasing", respectively. 
Counterexample 8. There exists a mil $\left(X_{n}\right)$ and some $\left(\tau_{n}\right) \in \mathcal{T}^{c}$ with $n \leq \tau_{n}+1 \leq \tau_{n+1},(n \in \mathbf{N})$, such that

$$
\lim _{m} P\left(\left\|D X\left(\tau_{2 m-1}, \tau_{2 m}\right)\right\|=1\right)=1 .
$$

Hence $\left(X_{\tau_{n}}\right)$ is not even a game fairer with time. However,

$$
\lim _{n} \sup _{m \geq n} P\left(\max _{q \in \mathbf{N}\left(n, \tau_{m}\right)}\left\|D X\left(q, \tau_{m}\right)\right\|=0\right)=0
$$

and hence $\left(X_{n}\right)$ is a $\left\{\tau_{n}\right\}$-mil.

1. Construction of Example 1. Let $\left([0,1), \mathcal{B}_{[0,1)}, P\right)$ be the Lebesgue probability space on $[0,1)$, (where $\mathcal{B}_{[0,1)}$ is the completion of the Borel $\sigma$-field w.r.t. the Lebesgue measure $P$ ). For $m \geq 1$, let $a_{m}=\prod_{j \leq m} 2^{j}$ and $Q_{m}$ the partition of $[0,1)$ in $a_{m}$-intervals of equal length. For $n=2 m-1$, let $\left(\mathcal{A}_{n}\right)$ be the $\sigma$-field generated by $Q_{m}$. Define $W_{n}$ as follows. For an interval $I$ of $Q_{m-1}$ set $W_{n}=2^{m}$ on the first interval of $Q_{m}$ that is contained in $I$ and $W_{n}=0$, elsewhere. Similarly, for $n=2 m$ let $\mathcal{A}_{n}=\sigma-\left\{Q_{m}\right\}$, and define $W_{n}$ in such a way that $W_{n}=2^{m}$ on the last interval of $Q_{m}$ which is contained in $I$ and $W_{n}=0$, elsewhere. It is easy to see that, for any $n, m \in \mathbf{N}$ with $n<2 m-1$, we have $W_{n}(2 m)=1=W_{n}(2 m-1)$. It follows that $\left(W_{n}\right)$ is neither an $\mathbf{N}$-sequential mil nor a game fairer with time, since $W_{n}$ converges to zero a.s.

But on the other hand, if we take the increasing cofinal sequence $\left(\tau_{n}\right)$ of nontrivial stopping times, given by $\tau_{m}=2 m-1$ on the set $\left\{W_{2 m-1}=0\right\}$ and $\tau_{m}=2 m$, elsewhere, then by the definition of $W_{n}$ it follows that $W_{r_{m}}=0$, for all $m \in \mathbf{N}$. Thus, for all $p, m \in \mathbf{N}$ with $p<\tau_{m}$, we have

$$
\max _{q \in \mathbf{N}\left(p, \tau_{m}\right)} D W\left(q, \tau_{m}\right)=\max _{q \in \mathbf{N}\left(p, \tau_{m}\right)}\left\|W_{q}\right\|, \text { a.s. }
$$

This with the a.s. convergence to zero of the sequence $\left(W_{n}\right)$ implies that $\left(W_{n}\right)$ must be a $\left\{\tau_{n}\right\}$-mil. Thus the example is well constructed.

2. Proof of Theorem 2. Let $\left(X_{n}\right),\left(\sigma_{n}\right)$ and $\left(\tau_{n}\right)$ be as supposed in the theorem. Then, by passing $\left(X_{\tau_{n}}\right)$ and $\left(X_{\sigma_{n}}\right)$ to subsequences, one can suppose (for simplicity of the proof) without any loss of generality that, for every $n \in \mathbf{N}$ we have $n \leq \tau_{n} \leq \sigma_{n}^{(-)}$. Now assume on the contrary that $X_{n}$ does not go to zero a.s. Then there exists $a>0$ and a set $A \in \mathcal{A}$ with $P(A)>0$ such that, for all $\omega \in A$, we have

$$
\lim \sup _{n}\left\|X_{n}(\omega)\right\|>5 a / 4
$$

We make the following claim.

For every $n_{1} \in \mathbf{N}$ and $0<\varepsilon<P(A) / 4$ there exists $n_{2} \in N\left(n_{1}\right)$ such that, for each $D \in \mathcal{A}_{\sigma_{n_{1}}}$ with $P(D)<P(A) / 4$ and each $n \in \mathbf{N}\left(n_{2}\right)$, there exists a set $M \in \mathcal{A}_{\sigma_{n_{2}}}$ with $M \cap D=\varnothing$ and $P(M)<\varepsilon$ such that

$$
\int_{M}\left\|X_{\sigma_{n}}\right\| d P \geq a P(A) / 4
$$


To prove the claim, let $n_{1} \in \mathbf{N}$ and $\varepsilon<P(A) / 4$ be given. By definition, one can find $P \in \mathbf{N}\left(\sigma_{n_{1}}\right)$ so large that, for all $n \in \mathbf{N}(p)$, we have

$$
P\left(\max _{q \in \mathbf{N}\left(p, \sigma_{n}\right)}\left\|D X\left(q, \sigma_{n}\right)\right\|>a / 4\right)<\varepsilon / 2 .
$$

First, by the property of the set $A$, there exists a strictly increasing finite sequence $\left\{p_{i}, 1 \leq l\right\}$ with $p<p_{1} \ldots<p_{l}$ such that, if we define for $i \leq 1$ the sets

$$
B_{i}=\left(\left\{\left\|X_{p_{i}}\right\|>5 a / 4\right\} \backslash \bigcup_{s<i}\left\{\left\|X_{p_{s}}\right\|>5 a / 4\right\}\right)
$$

and $B=\bigcup_{i \leq l} B_{i}$, then $B \in \mathcal{A}_{p_{l}}$ and $P(B)>15 P(A) / 16$.

Consequently, there exists a finite sequence $\left\{x_{j}^{*}, j \leq m\right\}$ of the unit ball of $F^{*}$ such that, for all $i \leq l$, we have $P\left(B_{i}^{1}\right)>P\left(B_{i}\right)-P(A) / 16 l$, where $F^{*}$ is the topological dual of $F$ and

$$
B_{i}^{1}=B_{i} \cap\left\{\max _{j \leq m}\left(x_{j}^{*}, X_{p_{i}}\right)>5 a / 4\right\} .
$$

Then $P\left(B^{1}\right)>7 P(A) / 8$, where $B^{1}=\bigcup_{i \leq l} B_{i}^{1}$.

On the other hand, since $\left(X_{\tau_{n}}\right)$ converges to zero a.s. there exists $k \in \mathbf{N}\left(p_{l}\right)$ such that if we set

$$
C=\left\{\left\|X_{\tau_{k}}\right\|>a / 4\right\}
$$

then $P(C)<\varepsilon / 2$.

Now define $n_{2}=k$ and let $D \in \mathcal{A}_{\sigma_{n_{1}}}$ with $P(D)<P(A) / 4$ and $n \in \mathbf{N}\left(n_{2}\right)$ be given. For every $i \leq 1$, set

$$
\begin{gathered}
H_{i}=\left\{\left\|D X\left(p_{i}, \sigma_{n}\right)\right\|>a / 4\right\}, \quad H=\bigcup_{i \leq l} H_{i}, \\
B_{i}^{2}=B_{i}^{1} \backslash\left(H_{i} \cup D\right) \text { and } B^{2}=\bigcup_{i<l} B_{i}^{2} .
\end{gathered}
$$

Then, by (6), $P(H)<\varepsilon / 2$ and $P\left(B^{2}\right)>7 P(A) / 8-3 P(A) / 8=P(A) / 2$. Further, by Proposition II.1.3 of J. Neveu [14], for every adapted sequence $\left(Z_{n}\right)$ in $L^{1}(F), s \in \mathbf{N}, \tau \in T(s)$ and $\sigma \in T\left(s, \tau^{(-)}\right)$we have

$$
Z_{\sigma}(\tau)=\sum_{q=s}^{\tau^{(-)}} I_{\{\sigma=q\}} Z_{q}(\tau) .
$$

Hence,

$$
\|D Z(\sigma, \tau)\| \leq \max _{q \in \mathbf{N}(s, \tau)}\|D Z(q, \tau)\|, \text { a.s. }
$$

(This fact will be applied also to the proof of Theorem 4.) 
This together with (6) implies that $P(G)<\varepsilon / 2$, where

$$
G=\left\{\left\|D X\left(\tau_{k}, \sigma_{n}\right)\right\|>a / 4\right\} .
$$

Therefore, by setting $S=C \bigcup G$ one gets $P(S)<\varepsilon$. Then, by taking $M_{i}=B_{i}^{2} \cap S, i \leq 1$, and $M=\bigcup_{i \leq l} M_{i}$, the set $M \in \mathcal{A}_{\tau_{k}}, P(M) \leq P(S)<\varepsilon$ and $M \cap D=\varnothing$. We show now that $M$ satisfies (5). To see this, for every pair $i \leq 1$ and $j \leq m$, let

$$
\left.Q_{i j}=B_{i}^{2} \cap\left\{x_{j}^{*}, X_{p_{i}}\right)>5 a / 4\right\} .
$$

Then it is evident that, for every $i \leq l,\left\{Q_{i j}, j \leq m\right\}$ is a partition of $B_{i}^{2}$. Hence $\left\{Q_{i j}, i \leq l, j \leq m\right\}$ forms a partition of $B^{2}$. Moreover, since $Q_{i j} \cap H_{i}=\varnothing$ and $Q_{i j} \in \mathcal{A}_{p_{i}}$ then on $Q_{i j}$ we have

$$
\left(x_{j}^{*}, X_{p i}\left(\sigma_{n}\right)\right) \geq\left(x_{j}^{*}, X_{p i}\right)-a / 4 \geq 5 a / 4-a / 4=a
$$

and

$$
\int_{Q_{i j}}\left(x_{j}^{*}, X_{p i}\right) d P=\int_{Q_{i j}}\left(x_{j}^{*}, X_{p i}\left(\sigma_{n}\right)\right) d P \geq a P\left(Q_{i j}\right) .
$$

Similarly, since $\left(Q_{i j} \backslash S\right) \cap C=\varnothing$ and $\left(Q_{i j} \backslash S\right) \in \mathcal{A}_{\tau_{k}}$, then on $Q_{i j} \backslash S$ we have $\left(x_{j}^{*}, X_{p i}\left(\sigma_{n}\right)\right) \leq\left(x_{j}^{*}, X_{p i}\right)+a / 4 \leq a / 4+a / 4=a / 2$ and

$$
\int_{Q_{i j} \backslash S}\left(x_{j}^{*}, X_{\sigma_{i}}\right) d P \leq a P\left(Q_{i j}\right) / 2 .
$$

Combining this with (9) we obtain

$$
\int_{M_{i}}\left(x_{j}^{*}, X_{\sigma_{i}}\right) d P \geq a P\left(Q_{i j}\right) / 2 .
$$

Thus, by summation over all $i \leq l$ and $j \leq m$, we get (5) and the claim is established.

Returning to the proof of the theorem, we construct by induction an increasing sequence $\left(n_{p} ; p \in \mathbf{N}\right)$ with the following property: whenever $D \in \mathcal{A}_{\sigma_{n_{p}}}$ with $P(D)<P(A) / 4$ and $n \geq n_{p+1}$ there exists $M \in \mathcal{A}_{\sigma_{n_{p+1}}}$ with $P(M)<2^{-(p+1)} P(A)$, $M \cap D=\varnothing$ and $\int_{M}\left\|X_{\sigma_{n}}\right\| d P \geq a P(A) / 4$. Thus given $p \in \mathbf{N}$ and $n \geq n_{p}$ we can construct, by finite induction for $i \leq p$, disjoint sets $D_{i}$ with $D_{1}=\varnothing, P\left(D_{i}\right)<$ $2^{-(i+1)} P(A)$ and $\int_{D_{i}}\left\|X_{\sigma_{n}}\right\| d P \geq a P(A) / 4$, for all $i \leq p$. Then $\int_{D}\left\|X_{\sigma_{n}}\right\| d P \geq$ $(p-1) a P(A) / 4$, where $D=\bigcup_{i \leq p} D_{i}$. This implies that $\lim _{n} E\left(\left\|X_{\sigma_{n}}\right\|\right)=\infty$, contradicting the condition (1). This completes the proof.

3. Proof of Theorem 4. Let $\left(X_{n}\right)$ be as supposed in the theorem. Then, as in the proof of Theorem 2, one can suppose without any loss of generality that $\left(X_{n}\right)$ is a $\left\{\sigma_{n}\right\}$-mil, for some strongly increasing $\left(\sigma_{n}\right) \in \mathcal{T}^{c}$. Thus, if we define the sequence $\left(Y_{n}\right)$ by $Y_{n}=X_{\sigma_{n}},(n \in \mathbf{N})$, then by (7) and the definition of $\left\{\sigma_{n}\right\}$-mils, it follows that $\left(Y_{n}\right)$ is a mil w.r.t. the increasing sequence $\left(\mathcal{B}_{n}\right)$ of sub- $\sigma$-algebras, given by $\mathcal{B}_{n}=\mathcal{A}_{\sigma_{n}}$, $(n \in \mathbf{N})$, with 


$$
\lim \inf _{n} E\left(\left\|Y_{n}\right\|\right)=\lim \inf _{n} E\left(\left\|X_{\sigma_{n}}\right\|\right)<\infty .
$$

Consequently, by the proof of Theorem 8 of [18], $\left(Y_{n}\right)$ can be written in a unique form:

$$
Y_{n}=W_{n}+Z_{n},(n \in \mathbf{N})
$$

where w.r.t. $\left(\mathcal{B}_{n}\right),\left(W_{n}\right)$ is a uniformly integrable martingale and $\left(Z_{n}\right)$ is a mil that goes to zero a.s. Here, it should be noted that in Theorem 8 of [18] M. Talagrand only claimed that the martingale $\left(W_{n}\right)$ is $L^{1}$-bounded. But in fact, he proved that if we define $h=\lim \inf _{n}\left\|Y_{n}\right\|$, a.s. then $h$ is integrable and for every $n \in \mathbf{N}$ we have $\left\|W_{n}\right\| \leq E^{n}(h)$ a.s. This implies that $\left(W_{n}\right)$ must be uniformly integrable and only its uniform integrability guarantees the uniqueness of (10). Returning to the proof, for every $n \in \mathbf{N}$, let us define $M_{n}=W_{n}\left(t_{n}\right)$, where $t_{n}=\min \left\{k \in N ; n \leq \sigma_{k}\right\}$, and put $P_{n}=X_{n}-M_{n}$. Then it is clear that, by the definitions of $\left(Y_{n}\right),\left(M_{n}\right),\left(P_{n}\right)$ and the properties of the decomposition (10), it follows that $\left(M_{n}\right)$ is a uniformly integrable martingale with $M_{\sigma_{n}}=W_{n},(n \in \mathbf{N})$ and $\left(P_{n}\right)$ is a $\left\{\sigma_{n}\right\}$-mil, satisfying the condition $\lim \inf _{n} E\left(\left\|P_{\sigma_{n}}\right\|\right)<\infty$ and such that the sequence $\left(P_{\sigma_{n}}=Z_{n}\right)$ converges to zero a.s. This together with Theorem 2 implies that $\left(P_{n}\right)$ also converges to zero a.s. It proves the decomposition (4) in the theorem, taking into account that any decomposition like (4) is always unique.

Finally, the conclusion of the theorem follows from Decomposition (4) and Chaterji's results Proposition 4.4. of [3] and Theorem 6 of [2] respectively. It completes the proof.

4. Construction of Example 7 and Counterexample 8. We shall construct large classes of Examples 7 and Counterexamples 8. Indeed, let $f$ be an arbitrary but fixed strictly increasing function from $\mathbf{N}$ to $\mathbf{N}$. Then $f(m) \geq m$, for all $m \in \mathbf{N}$. Let $([0,1)$, $\left.\mathcal{B}_{[0,1)}, P\right)$ and $Q_{k}$ with $k \geq 0$ be respectively the Lebesgue probability space and the partition of $[0,1)$ into $2^{k}$ intervals of equal length. For $m=0$, set $a_{0}=0=b_{0}$. For $m \in \mathbf{N}$, define $a_{m}=a_{m-1}+f(m)$ and $b_{m}=b_{m-1}+2^{a_{m-1}}$. Then, every $n \in \mathbf{N}$ can be written in a unique way $n=b_{m-1}+j$, with some $m \in \mathbf{N}$ and $1 \leq j \leq 2^{a_{m-1}}$. For arbitrary but fixed $n \in \mathbf{N}$ with the decomposition above, take $\mathcal{A}_{n}=\sigma-\left\{Q_{a_{m-1}+f(m)}\right\}$ and first define the real-valued random variable $Z_{n}$ on $[0,1)$ as follows. On the $j$-th interval $I_{j}^{(m-1)}$ of $Q_{a_{m-1}}$, set either $Z_{n}=2^{f(m)}$ or $Z_{n}=-2^{f(m)}$, respectively on the first interval of $Q_{a_{m}}$ that is contained in $I_{j}^{(m-1)}$, if either $j=2 p-1$ or $j=2 p$, respectively, with $1 \leq j \leq 2^{-1+a_{m-1}}$ and $Z_{n}=0$, elsewhere. Then, by the property of $f$, it is easy to check that, constructed in such a way, the sequence $\left(Z_{n}\right)$ still converges a.s. and in $L^{1}$ simultaneously. Thus, by Theorem [18], $\left(Z_{n}\right)$ is a mil. But with this construction $\left(Z_{n}\right)$ is not a universal mil. To see this, let us define the increasing cofinal sequence $\left(\tau_{n}\right)$ as follows. For every $m \in \mathbf{N}$, set $\tau_{m}=n$ on $I_{j}^{(m-1)}$, for $1 \leq j \leq 2^{a_{m-1}}$. Then it is not hard to check that $b_{m-1}<\tau_{m}$ and $P\left(\left\|Z_{b_{m-1}}\left(\tau_{m}\right)\right\|=1\right)=1$. This with the a.s. convergence to zero of $\left(Z_{n}\right)$ implies that $\left(Z_{n}\right)$ cannot be a universal mil. This completes the proof of the first example.

To construct a class of Counterexamples 8 we proceed as in the construction of Example 7. Indeed, let $a_{0}=0=b_{0}, a_{m}=a_{m-1}+f(m) 2^{a_{m-1}}, Q_{a_{m-1}}$ the partition $\left\{I_{j}^{(m-1)}, 1 \leq j \leq 2^{a_{m-1}}\right\}$ of $[0,1)$ into $2^{a_{m-1}}$ intervals of equal length. Given $1 \leq j \leq 2^{a_{m-1}}$, let $\left\{I_{j, k}^{(m-1)}\right\}, 1 \leq k \leq 2^{(j-1) f(m)}$, denote the partition of $I_{j}^{(m-1)}$ into $2^{(j-1) f(m)}$ intervals of 
equal length. For any fixed $n \in \mathbf{N}$ with the decomposition above, let us define $\mathcal{A}_{n}=\sigma-\left\{Q_{a_{m-1}+j f(m)}\right\}$ and the real-valued function $X_{n}$ on $[0,1)$ as follows. On $I_{j, k}^{(m-1)}$ set $X_{n}=2^{f(m)}$ or $X_{n}=-2^{f(m)}$, respectively, on the first interval of $Q_{a_{m-1}+j f(m)}$ that is contained in $I_{j, k}^{(m-1)}$, if $k=2 p-1$ or $k=2 p$, respectively, with $1 \leq p \leq 2^{(j-1) f(m)-1}$. Then, by the construction and the property of $f,\left(X_{n}\right)$ converges to zero a.s. and in $L^{1}$, and $\left(X_{n}\right)$ is a mil. Now, let us define the increasing cofinal sequence $\left(\tau_{n}\right)$ of bounded stopping times as follows. For $m \in M$, set $\tau_{2 m-1}=n-1$ and $\tau_{2 m}=n$ on $I_{j}^{(m-1)}$ with $n=b_{m-1}+j, 1 \leq j \leq 2^{a_{m-1}}$. Then it is easy to check that $n \leq \tau_{n}+1 \leq \tau_{n+1}$ and

$$
\lim _{k} \sup _{n \geq k} P\left(\max _{q \in \mathbf{N}(k, n)}\left\|D X\left(q, \tau_{n}\right)\right\|=0\right)=0
$$

but

$$
\lim _{m} P\left(\left\|D X\left(\tau_{2 m-1} \tau_{2 m}\right)\right\|\right)=1 .
$$

It proves that the mil $\left(X_{n}\right)$ and the sequence $\left(\tau_{n}\right)$ of bounded stopping times satisfy all the requirements of the counterexample. This completes the construction.

Acknowledgements. The author is indebted to Professors Cz. Ryll-Nardzewski and K. Musial for very pleasant and useful discussions during the author's stay at the Technical University of Wrocław, Poland, 1995-1997. Especially, he would like to express many thanks to the referee for helpful suggestions.

\section{REFERENCES}

1. L. H. Blake, A generation of martingales and two consequent convergent theorems, Pacific J. Math. 35 (1970), 279-283.

2. S. D. Chatterji, Martingale convergence and the Radon-Nikodym theorem in Banach spaces, Math. Scand. 22 (1968), 21-41.

3. S. D. Chatterji, Vector-valued martingales and their applications, in Probability in Banach spaces, Lecture Notes in Math. No 526 (1976), 33-51. 606.

4. A. Dvoretzky and A. Bellow, On martingales in the limit, Ann. Probab. 8 (1980), 602-

5. G. A. Edgar and L. Sucheston, Stopping times and directed processes, Encyclopedia of Math. and it's Appl. 47 (Cambridge University Press, 1992).

6. L. Egghe, Strong convergence of pramarts in Banach spaces, Canad. J. Math. 33 (1981), 357-361.

7. D. Q. Luu, Application of set-valued Radon-Nikodym theorem to convergence of multi-valued $L^{1}$-amarts, Math. Scand. 54 (1984), 101-113.

8. D. Q. Luu, Representation theorems for multi-valued (regular) $L^{1}$-amarts, Math. Scand. 58 (1986), 5-22.

9. D. Q. Luu, Decompositions and limits for martingale-like sequences in Banach spaces, Acts Math. Vietnam, 13 (1) (1998), 73-78.

10. D. Q. Luu, Convergence and lattice properties of a class of martingale-like sequences, Acta. Math. Hungar. 59 (3-4) (1992), 273-281.

11. D. Q. Luu, On further classes of martingale-like sequences, Probab. Theory and Appl. 37 (1992), 428-434.

12. A. Millet and L. Sucheston, Convergence of classes of amarts indexed by directed sets, Canad. J. Math. 32 (1980), 86-125. 541.

13. A. Mucci, Another martingale convergence theorem, Pacific J. Math. 64 (1976), 539- 
14. J. Neveu, Discrete parameter martingales, North-Holland Math. Library (1975).

15. M. Peligrad, A limit theorem for martingale-like sequence, Rev Roumaine Math. Pures Appl. 21 (1976), 733-736.

16. M. Slaby, Strong convergence of vector-valued pramarts and subramarts, Probab. Math. Statis. 5 (1985), 187-196.

17. S. Subramanian, On a generalization of martingales due to Blake, Pacific. J. Math. 48 (1985), 275-278.

18. M. Talagrand, Some structure results for martingales in the limit and pramarts, Ann. Probab. 13 (1985), 1192-1203.

19. Z. P. Wang and X. H. Xue, On convergence of vector-valued mils directed by a directed set in Proceedings International Conference: Almost everywhere convergence in probability and ergodic theory (Columbus, Ohio, 1988), 405-416. 